\title{
LC Headings from November 2019-February 2020 Lists
}

compiled by Ann Heinrichs

The new headings listed here reflect the most recent information available at the time of publication. Items in this list were selected from 2019 list numbers 11 (November 18) and 12 (December 16) and 2020 list numbers 01 (January 17) and 02 (February 14).

\section{SUBJECT HEADINGS}

150 Academic libraries--Activity programs [May Subd Geog] [sp2019103304]

450 UF Activity programs in academic libraries

550 BT Activity programs in education

150 Acquisition of data sets [May Subd Geog] [sp2019102623]

053 Z692.D37

$550 \quad$ BT Data sets

151 Acre (Israel)--History--Siege, 1189-1191 [sp2019102363]

$550 \quad$ BT Crusades--Third, 1189-1192

550 BT Sieges--Israel

150 Apostasy--Islam [sp 85006049]

450 UF Takfīr (Islam) DELETE FIELD

150 Bahai Faith and politics [May Subd Geog] [sp2019103115]

450 UF Bahai Faith--Political aspects

$450 \quad$ UF Politics and Bahai Faith

550 BT Political science

150 Book clubs (Discussion groups) in academic libraries [May Subd Geog] [sp2019103303] 550 BT Academic libraries--Activity programs

150 Catechisms, Urdu [sp2019103436]

450 UF Urdu catechisms

150 Christian shrines--New York (State) [sp2019102914]

150 Church buildings--Wales [sp2020000237]

150 Church work with autistic people [May Subd Geog] [sp2019000537] 550 BT Autistic people 
150 Church work with Generation X [May Subd Geog] [sp2019000536] 550 BT Generation X

150 Civilization, Western--Christian influences [sp2019102404] 550 BT Christian civilization

150 Climatology--Religious aspects-Islam [sp2019103302]

150 Column of Antoninus Pius (Vatican Palace, Vatican City) [sp 93006510] 450 UF Antonina, Colonna (Vatican Palace, Vatican City) ADD FIELD 450 UF Antonini Pii, Columna (Vatican Palace, Vatican City) ADD FIELD 450 UF Antonio Pio, Colonna (Vatican Palace, Vatican City) ADD FIELD 450 UF Colonna Antonina (Vatican Palace, Vatican City) ADD FIELD

150 Conflict management in the Qur'an [sp2019102655]

150 Drinking in rabbinical literature [Not Subd Geog] [sp2019102410] 053 BM496.9.D75

550 BT Rabbinical literature

150 Entertainment events--Religious aspects [sp2019102935

150 Entertainment events--Religious aspects--Christianity [sp2019102936]

150 Female friendship--Religious aspects--Society of Friends [sp2019102931]

150 Harmony (Philosophy)--Religious aspects--Buddhism [sp2019102810]

150 Hinduism and art [May Subd Geog] [sp2016002294] 450 UF Art and Hinduism

150 Hindustani music--Religious aspects--Hinduism [sp2019103047]

150 Inochentism [May Subd Geog] [sp2019001864] 053 BT1477

450 UF Innocentites

550 BT Christian heresies--History--Modern period, 1500-
150 Irreecha (Festival) [May Subd Geog] [sp2019101403] 450 UF Irreechaa (Festival) 450 UF Irreessa (Festival)

550 BT Oromo (African people)--Rites and ceremonies

150 Islam and poetry CHANGE HEADING

150 Poetry--Religious aspects-Islam [sp 85068419] $450 \quad$ UF Islam and poetry [Former heading]

150 Islamic clothing and dress--Law and legislation [May Subd Geog] [sp2019102354]

$150 \quad$ Jewface [May Subd Geog] [sp2019101411] 450 UF Jew-face

550 BT Impersonation

680 Here are entered works on the caricature of Jews, generally by non-Jews, through the use of makeup, mannerisms, speech patterns, etc.

150 Jewish religious poetry, Judeo-Italian ～[May Subd Geog] [sp2019102320] 450 UF Judeo-Italian Jewish religious poetry

550 BT Judeo-Italian poetry

150 Judeo-Italian literature [May Subd Geog] [sp2019101432] 551 BT Italy--Literatures

150 Judeo-Italian poetry [May Subd Geog] [sp2019102319] 550 BT Judeo-Italian literature

150 Library linked data [May Subd Geog] [sp2019102475] 053 Z666.73.L56

550 BT Library metadata

550 BT Linked data

150 Mina (Sect) [May Subd Geog] [sp2019102903]

053 BL2018.7.M56

450 UF Chota Mela (Sect)

$550 \quad$ BT Sikh sects

50 Mizrahim [May Subd Geog] [sp 85070504]

450 UF Adot HaMizrach ADD FIELD

450 UF 'Adot ha-Mizrah ADD FIELD

450 UF Mizrachim ADD FIELD

150 Mormon Church [May Subd Geog] [sp 85087285] 550 RT Mormons DELETE FIELD 
150 Mormons [May Subd Geog] [sp 85087296]

053 BX8601-BX8695 ADD FIELD

550 RT Mormon Church DELETE FIELD

550 BT Christians ADD FIELD

150 Mothers--Death--Religious aspects [sp2019102933]

150 Mothers--Death--Religious aspects--Christianity [sp2019102932]

150 Multiple religious belonging [May Subd Geog] [sp2018002528 550 BT Identification (Religion)

150 Odors--Religious aspects [sp2019103296]

150 Odors--Religious aspects--Judasim ～[sp2019103295]

150 Open educational resources [May Subd Geog] [sp2019000514] 053 LB3045.74 ADD FIEL

450 UF OERs (Open educational resources) ADD FIELD

150 Open educational resources in libraries [May Subd Geog] [sp2019103075] 450 UF Libraries--Special collections--Open educational resources $\begin{array}{ll}450 & \text { UF Libraries } \\ 550 & \text { BT Libraries }\end{array}$

150 Other (Philosophy)--Religious aspects--Hinduism [sp2019102702

150 Other (Philosophy)--Religious aspects-Jainism [sp2019102703]

150 Paz, Nuestra Señora de la [Not Subd Geog] [sp2019103138]

450 UF Mother of Peace

450 UF Nuestra Señora de la Paz

450 UF Our Lady of Peace

450 UF Our Lady Queen of Peace

450 . UF Paz, Reina de

450 UF Peace, Mother of

450 UF Peace, Our Lady of

450 UF Peace, Queen of

450 UF Reina of Peace

$500 \quad$ BT Mary Blessed Virgin, Saint--Apparitions and miracles

500 BT Mary, Blessed Virgin, Saint--Devotion to
150 Peace in rabbinical literature [sp2016000296] 053 BM496.9.P43

550 BT Rabbinical literature

150 Poetry--Religious aspects--Anabaptists [sp2019101448]

150 Poetry--Religious aspects--Buddhism [sp2019101446]

150 Poetry--Religious aspects--Christianity [sp2019101442]

150 Poetry--Religious aspects--Hinduism [sp2019101447]

150 Poetry--Religious aspects--Unification Church [sp2019101449]

150 Religion and poetry CHANGE HEADING

Poetry--Religious aspects DELETE GEOG [sp 85112576 450 UF Religion and poetry [Former heading

150 Religious drama, Chinese [May Subd Geog] [sp2019102969] 450 UF Chinese religious dram

550 BT Chinese drama

150 Sharqāwīyah ～[May Subd Geog] [sp2019103255]

450 UF Sharkāwa

450 UF Sharqāwa

450 UF Sherkāâ

150 Shrine of Saint Elizabeth Ann Seton (New York, N.Y.) [May Subd Geog] ～[sp2019102916] $450 \quad$ UF Saint Elizabeth Seton Shrine (New York, N.Y.)

450 UF Seton Shrine (New York, N.Y.)

450 Shrine or Blessed Elizabeth Seton (New York, N.Y.)

450

450 UF St.

150 Sikh Americans CANCEL HEADING [sp 90004207]

682 This authority record has been deleted because the heading is covered by the subThis authority record has been deleted because the

150 Sikhs--United States [sp 90004208$]$

450 UF Sikh Americans [Former heading] ADD FIELD 
150 Sufi literature, Bosnian [May Subd Geog] [sp2019103181]

$450 \quad$ UF Bosnian Sufi literature

550 BT Bosnian literature

150 Takfîr (Islam) [Not Subd Geog] [sp2019103445]

053 BP190.5.T35

450 UF Excommunication (Islam)

450 UF Takfeer (Islam)

550 BT Islam

151 Tiberias, Lake (Israel)--In the Bible CHANGE HEADING

151 Galilee, Sea of (Israel)--In the Bible [sp 91004054 ]

450 UF Tiberias, Lake (Israel), in the Bible [Former heading]

150 Veterinary medicine--Religious aspects [sp2019102737]

150 Veterinary medicine--Religious aspects--Christianity [sp2019102736]

150 Women fakirs [May Subd Geog] [sp2016002109]

$550 \quad$ BT Fakirs

150 Yashan (Jewish law) [Not Subd Geog] [sp2019000191]

053 BM523.5.Y37

450 UF Vintage grain (Jewish law)

450 UF Yoshon (Jewish law)

550 BT Jews--Dietary laws

\section{GENRE/FORM TERMS}

155 Avadana stories [gp2019026109]

455 UF Apadana stories

455 UF Apadana tales

455 UF Avadana tales

455 UF Stories, Apadana

455 UF Stories, Avadana

455 UF Tales, Apadana

455 UF Tales, Avadana

555 BT Biographies

555 BT Literature

680 Stories that are usually narrated by the Buddha and that identify characters in the past as former births of characters in the present. 